\title{
Two different methods of cleaning and disinfection of endoscopy study of terminal disinfection
}

\author{
Yan-Ming Li ${ }^{1, a}$, Yi-An Lü ${ }^{2, b}$ \\ ${ }^{1}$ Nursing College Beihua University, Jilin, 132013, China \\ ${ }^{2}$ Clinical Medical College Beihua University, Jilin, 132013, China \\ a843789777@qq.com, ${ }^{\text {b4 } 492964574 @ q q . c o m ~}$
}

\begin{abstract}
Keywords: Gastroscope, Cleaning and disinfection, Modified method, Sterilization qualified rate. Abstract. 200 sets of used gastroscopes for gastroscopy were randomly divided into group A and B, 100 sets in each group. Gastroscopes in group A were cleaned and disinfected with the conventional method and those in group B were cleaned and disinfected with a modified cleaning and disinfection method. On the basis of the conventional method, the modified cleaning and disinfection method was supplemented a booster pump and a cleaning spray gun to replace the syringe for the suction-infusion in the conventional method, and the sterilization effects of two methods were studied and compared. The results showed that the gastroscope sterilization qualified rate achieved with the modified cleaning and disinfection method was $98 \%$, that with the conventional cleaning and disinfection method was $78 \%$, and there was a statistically significant difference in the qualified rate between the two groups $(p$ $<0.05)$; the incidence of complete and incomplete air/water pipes of gastroscopes in group B was significantly lower than that in the control group (group A) $(p<0.05)$. The modified method for the cleaning and disinfection of gastroscopes shows a better disinfection effect, lower cost and strong practicability, worthy of clinical popularization.
\end{abstract}

\section{Introduction}

With its development and popularization, endoscopic technology has been used more and more widely as an invasive diagnostic and therapeutic technology in clinic, but the nosocomial infection risk caused by its application has increased [1]. Due to the particularity in the operation of endoscope, such as the exposure to blood, body fluids, mucous membrane and tissues of the body, the delayed cleaning and disinfection, or improper disinfection methods after use, would lead to the residue of organic matter and microorganisms, and even the formation of biofilms, which can result in the failure of endoscope cleaning and disinfection, eventually the occurrence of nosocomial infection, so that how to clean and disinfect the endoscope in the process of its application is very important. Gastroscopy is an important way for the diagnosis and treatment for gastrointestinal diseases, but due to characteristics in the complexity of its internal structure and the particularity of its materials, it is more difficult to clean and sterilize the gastroendoscope after use, and meet the requirements for the full sterilization of it, then leading to an iatrogenic cross-infection [2]. For this reason, a modified cleaning and disinfecting method was used for the sterilization of gastroscope, and a strengthened hanging disinfection method was adopted to maintain the disinfection in our hospital, for ensuring a high-level disinfection of gastroscope and achieving an ideal effect to prevent the cross-infection.

\section{Materials and Methods}

Materials. The section headings are in boldface capital and lowercase letters. Second level headings are typed as part of the succeeding paragraph (like the subsection heading of this paragraph).

\section{Methods}

Convetional disinfection method. The used gastroscopes were washed manually with flowing water, soaked in a multi-enzyme cleaning agent, rinsed with flowing water, in which a syringe was used 
to pump and infuse the water, then soaked in $2 \%$ glutaraldehyde solution, and finally rinsed with distilled water.

\section{Modified disinfection method.}

Structures of the disinfection device. The bottom connector of multi-enzyme cleaning tank was installed a closable t-branch pipe (connecting sewer, booster pump and inlet pipe, respectively). The hydraulic pressure was $100-500 \mathrm{kPa}$, the hydraulic discharge was adjusted by the cleaning spray gun, and the rest connectors at the bottom of cleaning tank were all installed closable bipasses.

Disinfection methods. (1) Pre-cleaning: the used endoscopes were washed alternatively by tap water and gas through a suction apparatus for $1 \mathrm{~min}$ to remove the most of the contaminants on the surface of gastroscopes and within the lumen of them, and dredge the air/water hole of endoscopes. (2) Gastroscope cleaning disinfection by combined manual and electric method: $\square$ the gastroscopes were rinsed with flow water and cleaning gun for 2min; (2) the rinsed gastroscopes were soaked in a multi-enzyme agent and rinsed repeatedly in a pressed flushing way for $2 \mathrm{~min}$; $\square$ the gastroscopes were rinsed with flow water and cleaning gun for $2 \mathrm{~min}$ again to remove the residual detergent; (4) the rinsed gastroscopes were wiped dry with dried gauze, and then placed a disinfection tank containing $2 \%$ glutaraldehyde solution for $30 \mathrm{~min}$ for the immersion disinfection, and simultaneously the glutaraldehyde was injecteded into the cavity of gastroscopes; $\square$ the gastroscopes were rinsed with flow water and cleaning gun to remove the residual glutaraldehyde; (6) the sterilized gastroscopes were placed in a distilled water tank and flushed with distilled water for $2 \mathrm{~min}$. (3) Re-disinfection: the gastroscopes were hung and the gastroscopes in the hanging position were disinfected one more time. $10 \mathrm{ml}$ of $2 \%$ glutaraldehyde solution were injected into the cavity of gastroscopes, and then $100 \mathrm{ml}$ distilled water were injected into it; $2 \%$ glutaraldehyde solution was used to wipe the surface of gastroscopes 3 times and distilled water was used to clean it 6 times. The gastroscopes were wiped with ethanol 1 times when they were going to be used.

\section{Monitoring}

Monitor items. A cotton swab was used to sample by swabbing the surface surrounding the body of gastroscopes and at a distance of 20 30cm from the lens 12 times [3]; the sampling of the inner cavity of gastroscopes was conducted by rapidly injecting $10 \mathrm{ml}$ sterile saline into the cavity through the biopsy channel, then receiving the sterile saline that flowed out with a sterile beaker, and the received sterile saline was immediately send to the laboratory center of our hospital for the test of total and pathogenic bacteria.

Sampling time. After they were used for the diagnosis and treatment in patients, the used gastroscopes were grouped, cleaned and disinfected, and the samples were collected just before the reuse of them.

Monitoring indicators. It was considered to be qualified in the sterilization that the total bacteria detected were $\leq 20 \mathrm{CFU} /$ set and no pathogenic bacterium was detected after the disinfection of gastroscopes [4].

Statistical Analysis. All data were statistically analyzed using SPSS17.0 software. The count data were tested with $x^{2}$ test and the measurement data were tested with $t$-test. $P<0.05$ was considered a significant difference in statistics.

\section{Results}

Comparison on Disinfection Effects of Gastroscopes between Two Groups. As shown in Table 1, the sterilization qualified rate of gastroscopes disinfected with the modified disinfection method was significantly higher than that with the conventional disinfection method $(p<0.05)$.

Pipe Blockage Incidence of Gastroscopy Operation. The complete and incomplete blockage incidence of gastroscope air/water pipes in the modified group was significantly lower than that in the conventional group $(p<0.05)$. The results are shown in Table 2. 
Table 1 Sterilization qualified rates of gastroscopes in twogroups (\%)

\begin{tabular}{lccc}
\hline Gorups & Gastroscopes $(n)$ & Qualified number $(n)$ & Qualified rates \\
\hline Conventional & 100 & 78 & 78.0 \\
Modified & 100 & 98 & 98.0 \\
\hline $\mathrm{x}^{2}$ & & & 3.78 \\
$p$ & & & 0.006 \\
\hline
\end{tabular}

Table 2 Complete and incomplete blockage incidences of gastroscope air/water pipes

\begin{tabular}{lccc}
\hline Groups & Gastroscopes $(n)$ & Blockage number & Blockage incidence(\%) \\
\hline Conventional & 100 & 9 & 9.0 \\
Modified & 100 & 1 & 1.0 \\
\hline $\mathrm{x}^{2}$ & & 3.75 \\
$p$ & & 0.009 \\
\hline
\end{tabular}

\section{Discussion}

Endoscopy is an invasive diagnostic and therapeutic technology, and has been widely used in clinic for many years. Endoscopy can lead to infections by three ways, including patients themselves, medical personnel and other patients, which is a potential hazard for nosocomial infections. A foreign scholars' review [5]on infections induced by endoscopy showed that endoscopy caused 281 cases of pathogen infections, and causes of the infections were the lack of appropriate guideline and standards for the cleaning and disinfection of endoscopy, no compliance of the standards of endoscopy operation guide, the application of unqualified disinfectants or incomplete drying of the endoscopes. With the successive issue and the implementation of endoscopic operation guide specification, the iatrogenic infection caused by the endoscopy has significantly decreased [6].

Due to its delicate internal structure, special materials, heat intolerance, slender pipes, and easily adhering to blood, mucus, tissue fluid and excrement, pathogenic microorganisms tend to remain in endoscope and it is difficult to clean and sterilize the microorganisms remained in it after the use of it [7], leading to the difficulty to meet the requirements for the full sterilization. Therefore, it is necessary to fully clean and sterilize the used endoscope.

This study showed that the cleaning and disinfecting qualified rate of used gastroscopes cleaned and sterilized with the modified method was $98.0 \%$, and was significantly better than that with the conventional method $(78.0 \%)(p<0.05)$. The conventional manual method for the cleaning of gastroscopes with flowing water is too simple and less efficient, especially, due to the small suction-infusion power of syringe and its poor continuity, the decontamination is often incomplete [8]. In this study, a modified sterilization method was used, in which on the basis of conventional sterilization method, a booster pump and a cleaning spray gun were used to replace the syringe used for the suction-infusion to overcome the disadvantages of the conventional manual cleaning method. Moreover, more attention was paid to the secondary contamination in the hanging state to ensure a high level of comprehensive disinfection of gastroscopes and the safety of the application of gastroscopes in patient.

This study showed that with the modified method, there was only one air/water pipe of gastroscopy was completely or not completely blocked, but 9 air/water pipes were completely or not completely blocked when the conventional method was used, suggesting a statistically significant difference in the incidence of complete or incomplete blocking of pipes between the two groups $(p<0.05)$. Most problems occurred during the endoscopy used to be the blocking of air/water pipes, blood and 
secretions could adhere to the surface and the inner surface of various lumen of used gastroscope to form a protective film, preventing the penetration of disinfectants and resulting in the incomplete disinfection [9]. The application of the modified cleaning and disinfection method can completely clear away the stains within the lumen to improve the disinfection effect, and the method is with a lower cost and better practicability, and worthy of clinical popularization.

\section{References}

[1] Lingling Pan, Hong Xu, Xiaowei Zhao, Progress in the research on the cleaning and disinfection of gastroscope, J. Chinese Journal of Disinfection. 30 (2013) 252.

[2] Aijuna Feng, Analysis of 120 cases with portal hypertensive gastropathy in endoscopic features, J. Chongqing Medicine. 38 (2009) 409.

[3] Mingdong Zhou, Yuanping Yang, Mo Lihua, Different methods for pre-cleaning of gastroscope, J. Chinese Journal of Nosocomiology. 23 (2013) 620.

[4] Peng Yang, Yueyue Lu, Xuyang Zuo, Effect of modified cleaning and disinfection method of gastroscopes on prevention of iatrogenic cross infections caused by hepatitis B virus, J. Chinese Journal of Nosocomiology. 23 (2013) 618.

[5] Xiaolan Tang, Research progress in the cleaning and disinfection of digestive endoscope, J. Chinese Journal of Disinfection. 27(2010)603-605.

[6] Yuqin Shen, Lishu Xiao, Jianying $\mathrm{Hu}$, Comparison on effects of three different methods for the cleaning of surgical suction tubes, J. Journal of Nurses Training. 26 (2011) 1833-1835.

[7] Qing Gu, Ying Fang, Liying Gu, Endoscopic biopsy channels washed by endoscopic cleaning brush and disposable swab club: A comparison of their clearance effect, J. Chinese Journal of Nosocomiology. 20 (2010) 2630-2632.

[8] Chunyan Hao, Research progress in the prevention and control of catheter-related bloodstream infection, J. Journal of Nurses Training. 24 (2009) 2034-2035.

[9] Xiaoyi Li, Zhiying Jia, Zhongjun Feng, Cleaning and disinfection management for digestive endoscopes in 12 hospitals in Shijiazhuang: An investigation report, J. Chinese Journal of Nosocomiology. 19 (2009) 536-538. 